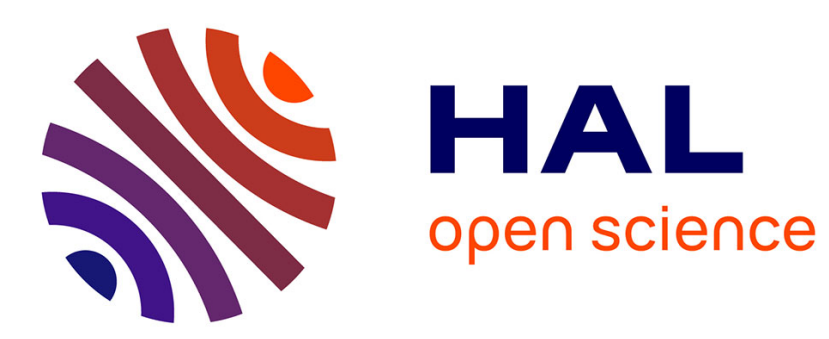

\title{
Les salles de musique à Paris : hiérarchies de légitimité et manières d'entendre les genres musicaux
}

Myrtille Picaud

\section{To cite this version:}

Myrtille Picaud. Les salles de musique à Paris : hiérarchies de légitimité et manières d'entendre les genres musicaux. Actes de la Recherche en Sciences Sociales, 2015, 206-207 (1), 10.3917/arss.206.0068 . halshs-01678061

\section{HAL Id: halshs-01678061 https://shs.hal.science/halshs-01678061}

Submitted on 8 Jan 2018

HAL is a multi-disciplinary open access archive for the deposit and dissemination of scientific research documents, whether they are published or not. The documents may come from teaching and research institutions in France or abroad, or from public or private research centers.
L'archive ouverte pluridisciplinaire HAL, est destinée au dépôt et à la diffusion de documents scientifiques de niveau recherche, publiés ou non, émanant des établissements d'enseignement et de recherche français ou étrangers, des laboratoires publics ou privés. 
Myrtille Picaud

Ecole des Hautes Etudes en Sciences Sociales

Centre Européen de Sociologie et de Science Politique

myrtille.picaud@hotmail.fr

\section{Les salles de musique à Paris : hiérarchies de légitimité et manières d'entendre les genres}

\section{musicaux $^{1}$}

[pre-print]

\section{Résumé}

Des lieux très différents (bars musicaux, théâtres, salles de concert, boîtes de nuit) forment l'espace des salles de musique à Paris. Ces dispositifs cadrent le lien entre artistes et publics et participent de la signification sociale conférée à la sortie musicale. A partir d'une analyse des correspondances multiples et d'une enquête ethnographique, cet article se propose d'observer la relation qui se construit entre offres de sortie et genres musicaux dans un cadre géographique spécifique. L'examen des principes qui structurent l'espace des salles révèle ainsi une inégale reconnaissance institutionnelle des manières de sortir et d'entendre la musique. Sur le plan de la renommée, des salles de taille importante occupent une position dominante, qui mène à des recoupements de genres en partie contre-intuitifs. L'importance de l'ancienneté des salles est également rendue visible.

\footnotetext{
${ }^{1}$ Cet article est issu d'une recherche effectuée dans le cadre du Partenariat Institutions-Citoyens pour la Recherche et l'Innovation dirigé par Stéphane Dorin, « Les publics de la musique classique au XXI ${ }^{\text {me }}$ siècle. Enquête sur les publics de la musique classique vivante et sa diffusion numérique en Ile-de-France et sur le plan national».
} 
Aujourd'hui comme hier, l'existence des salles de musique à différentes échelles participe à construire ou renforcer les représentations d'une ville en tant que « capitale culturelle $»^{2}$. Cependant, les salles de musique s'inscrivent dans un territoire dont l'étendue et les contours varient, non seulement en fonction de leur localisation, de leur reconnaissance et de leur architecture, mais aussi en fonction des artistes et des publics du lieu. Certaines salles sont visibles - c'est-à-dire aussi « entendues »-au niveau du quartier ${ }^{3}$, d'autres, de la ville, voire elles acquièrent une résonnance nationale ou internationale. Si une partie des salles parisiennes est fréquentée par des habitués des lieux environnants, d'autres recrutent leurs publics dans les départements limitrophes de la ville, en région, ou encore attirent des « touristes ».

Espaces centraux de la chaîne des intermédiaires du travail artistique ${ }^{4}$, les salles de musique articulent un dispositif matériel et une programmation, dont les professionnels ${ }^{5}$ se révèlent des « hommes doubles », situés à la « charnière du social et du culturel » ${ }^{6}$. Pierre Bourdieu évoque l'éditeur comme « celui qui a le pouvoir tout à fait extraordinaire d'assurer la publication, c'està-dire de faire accéder un texte et un auteur à l'existence (Öffentlichkeit), connue et reconnue $»^{7}$. De la même manière, la salle de musique fait monter sur scène les artistes et leurs œuvres. Plus fortement encore que le livre ou le disque, elle repose sur un agencement matériel et physique qui organise la rencontre entre des publics et des artistes dans un cadre

\footnotetext{
${ }^{2}$ Christophe Charle (dir.), Le temps des capitales culturelles, XVIII e-XXe siècles, Paris, Champ Vallon, 2009.

${ }^{3}$ Sur l'intérêt de réfléchir à l'échelle du quartier, voir notamment Mélanie Traversier, « Le quartier artistique, un objet pour l'histoire urbaine », Histoire urbaine, $\mathrm{n}^{\circ} 26,2009$, p. 5-20.

${ }^{4}$ Voir en particulier Wenceslas Lizé, Delphine Naudier et Olivier Roueff, Intermédiaires du travail artistique. À la frontière de l'art et du commerce, Ministère de la Culture et de la Communication, Département des études, de la prospective et des statistiques, 2011 ; et le numéro «Intermédiaires culturels, territoires professionnels et mobilisations collectives dans les mondes de l'art», Le Mouvement Social, n 243, 2013.

${ }^{5}$ Nous utiliserons ici le terme «programmateur » sans le féminiser afin de ne pas alourdir le propos. Dans la population que nous avons interrogée à Paris et Berlin, les programmateurs (37) sont plus nombreux que les programmatrices (12).

${ }^{6}$ Christophe Charle, «Le temps des hommes doubles », Revue d'histoire moderne et contemporaine, n 39,1992 , ,p. 75.

${ }^{7}$ Pierre Bourdieu, «Une révolution conservatrice dans l'édition », Actes de la recherche en sciences sociales, n $126-127,1999$, p. 3.
} 
géographique donné, constituant ainsi en partie les «conditions pérennes de la réception ${ }^{8}$. Comme le souligne Olivier Roueff, de tels intermédiaires sont centraux dans la formation et le fonctionnement d'un champ : «c'est essentiellement par leur médiation que sont construites et prescrites, aux auditeurs comme aux musiciens, les normes spécifiques du champ en gestation. Ce faisant, les intermédiaires sont en quelque sorte les "magiciens" de l'homologie structurale, c'est-à-dire des spécialistes de sa production puisque ses effets n'adviennent pas "magiquement" d'eux-mêmes. Au fil d'un intense travail collectif tantôt coopératif, tantôt concurrentiel d'ajustements, d'échecs répétés et de réussites exploitées, ils transforment des conditions sociales de possibilité virtuelles en une mise en correspondance pratique - et, en réalité, jamais parfaitement homologue, ce qui constitue l'un des ressorts de la dynamique de transformation des champs - entre l'espace des publics et l'espace des producteurs. ${ }^{9}$

Si la réception des œuvres n'est pas intégralement contrôlée par les intermédiaires, ils ont l'initiative de leur mise en rapport avec les consommateurs. Le dispositif de la salle influence également l'expérience du concert des artistes ${ }^{10}$. En cela, la «musique vivante » se distingue des pratiques d'écoute individuelles et privées, de manière plus ou moins marquée en fonction des genres musicaux. Des travaux récents examinent les correspondances entre le type de musique programmé, les lieux physiques et leur localisation, les manières d'écouter des publics ou encore le lien avec la structure urbaine ${ }^{11}$. D'autres privilégient l'étude des salles de concert ${ }^{12}$,

\footnotetext{
${ }^{8}$ Olivier Roueff, «Les homologies structurales : une magie sociale sans magiciens ? La place des intermédiaires dans la fabrique des valeurs », in Philippe Coulangeon, Julien Duval (eds), Trente ans après La Distinction, Paris, La Découverte, 2013, p. 160.

${ }^{9}$ Olivier Roueff, «"La montée des intermédiaires" Domestication du goût et formation du champ du jazz en France, 1941-1960 », Actes de la recherche en sciences sociales, $\mathrm{n}^{\circ}$ 181-182, 2010, p. 36.

${ }^{10}$ Marc Perrenoud, «Jouer "le jazz" : où, comment? Approche ethnographique et distinction des dispositifs de jeu », Sociologie de l'Art, n ${ }^{\circ}$, 2006, p. 25-42.

${ }^{11}$ Par exemple, Wenceslas Lizé, « "Le goût jazzistique en son champ" L'espace parisien de la jazzophilie », Actes de la recherche en sciences sociales, $\mathrm{n}^{\circ}$ 181-182, 2010, p. 60-87; Elsa Vivant, Le rôle des pratiques culturelles off dans les dynamiques urbaines, thèse de doctorat en urbanisme, Ecole doctorale Ville et Environnement, Université Paris 8, 2006.

${ }^{12}$ Hans Erich Bödeker, Patrice Veit, Michael Werner (dirs.), Espaces et lieux de concert en Europe, 1700-1920. Architecture, musique, société, Berlin, Berliner Wissenschafts-Verlag, 2008.
} 
l'assignation symbolique d'un genre musical à un territoire social et à un imaginaire urbain ${ }^{13}$ ou la reconstitution des trajectoires des agents et œuvres qui participent à l'établissement d'un espace artistique, comme le théâtre ${ }^{14}$. Le concept de «scène » musicale a également été mobilisé par la littérature sur la musique ${ }^{15}$. Si ses définitions varient, le terme renvoie à un ensemble d'agents, producteurs, intermédiaires et récepteurs de musique, qui s'agrègent autour d'un genre musical. Déterminant, l'ancrage géographique de la scène peut être considéré à l'échelle locale, translocale (plusieurs scènes locales reliées) ou virtuelle.

La définition et le classement des genres musicaux font l'objet de luttes qui mettent aux prises l'industrie de la musique, les artistes, la critique, les administrateurs culturels et les publics. Ce sont également des catégories d'analyse mobilisées dans l'appréhension des échelles de légitimité culturelle, opposant les musiques « savantes » aux musiques «populaires ».

A la suite des débats suscités par La Distinction de Pierre Bourdieu et par la thèse de Richard Peterson qui oppose l'omnivorisme des personnes de statut élevé à l'univorisme de ceux au bas de la hiérarchie sociale ${ }^{16}$, Philippe Coulangeon a suggéré la notion d'éclectisme pour analyser le phénomène de la légitimité culturelle ${ }^{17}$. L'appréciation simultanée de biens plus ou moins légitimes (musique classique et rock par exemple) serait une manière de se distinguer. Coulangeon souligne également que s'il existe bien un «éclectisme cultivé », celui-ci privilégiant certaines combinaisons de genres, les données font défaut quant à la « manière de consommer » ces objets culturels.

\footnotetext{
${ }^{13}$ Par exemple, dans le cas du rap, il s'agit de la «banlieue »; voir le chapitre « Rap et banlieue : le thème imposé », in Karim Hammou, Une histoire du rap en France, Paris, La Découverte, 2012, p. 70-90.

${ }^{14}$ Christophe Charle, Théâtres en capitales. Naissance de la société du spectacle à Paris, Berlin, Londres et Vienne, Paris, Albin Michel, 2008.

${ }^{15}$ Andy Bennett, Richard A. Peterson, Music scenes: local, translocal and virtual, Vanderbilt University Press, 2004; Gérôme Guibert, Scènes locales, scène globale. Contribution à une sociologie économique des producteurs de musiques amplifiées en France, Thèse de doctorat de sociologie Université de Nantes, 2004 ; Gérôme Guibert, " La notion de scène locale. Pour une approche renouvelée de l'analyse des courants musicaux ", in Stéphane Dorin (dir.), Sound Factory. Musique et Industrie, Paris, Seteun, 2012.

${ }^{16}$ Richard A. Peterson, « Understanding Audience Segmentation: From Elite and Mass to Omnivore and Univore $»$, Poetics, nº 4, 1992, p. 243-258.

${ }^{17}$ Philippe Coulangeon, «"La stratification sociale des goûts musicaux." Le modèle de la légitimité culturelle en question », Revue française de sociologie, $\mathrm{n}^{\circ} 1,2003$, p. 3-33.
} 
L'analyse présentée ici porte sur les lieux de sortie musicale à Paris et non sur les déclarations des goûts musicaux, recensées statistiquement à l'échelle nationale. Nous avons pris en compte l'ensemble des genres musicaux qui y sont représentés, en considérant que chaque « scène » existe dans un espace relationnel et que les genres se définissent les uns par rapport aux autres en un lieu géographiquement déterminé18. Il s'agit alors de construire l'espace des lieux de musique à partir d'un double critère : d'une part, les propriétés objectives des salles telles qu'elles apparaissent aux yeux des publics; d'autre part, le point de vue des agents qui y travaillent.

Les salles parisiennes sont nombreuses et morphologiquement très diversifiées. Ainsi, l'appréhension de ce qui constitue une salle de musique (à la différence d'une salle de concert) ne va pas de soi. La sélection des «salles » a donc été faite en prenant en considération l'existence d'une programmation de musique dite vivante suffisamment régulière (au moins 4 fois par mois). Si la différence entre animation musicale et concert ou performance est parfois ténue, nous avons choisi de prendre en compte les DJs (disc-jockey) et avons exclu la diffusion de musique enregistrée. Les lieux présentant des spectacles de cabaret musical, de transformisme, dans une configuration souvent en lien avec une restauration (Chez Michou) ou parfois avec des revues érotiques, ont été conservés si la musique était jouée sur scène (le Moulin Rouge par exemple n'a pas été retenu). Lieux amateurs et professionnels n'ont pas été départagés. Cela aurait pu induire une sousreprésentation d'espaces culturels moins légitimes ${ }^{19}$ et exclure certains genres, alors même que cette recherche se propose d'interroger les différentes manières dont se structure l'expérience de la musique vivante, sans les hiérarchiser a priori. Les espaces présentant des amateurs de manière publique (Maison des Pratiques Artistiques Amateurs, Centre Barbara Fleury la Goutte d'Or, mais aussi dans le cadre de bœufs ${ }^{20}$ ) ont donc été inclus. De même, nous avons choisi d'examiner des lieux de taille, statuts et orientations artistiques très différents.

\footnotetext{
${ }^{18}$ Ainsi que nous le verrons ensuite, $16 \%$ seulement des salles se spécialisent sur un seul genre.

${ }^{19}$ Vincent Dubois, Jean-Matthieu Méon, Emmanuel Pierru, Les mondes de l'harmonie. Enquête sur une pratique musicale amateur, Paris, La Dispute, 2009.

${ }^{20}$ Les termes «bœuf» ou «jam session» s'appliquent à des séances d'improvisation musicale auxquelles musicien.nes amateur.es et professionnel.les sont invité.es à participer. A Paris, ces séances ont lieu principalement dans des salles de jazz et de blues.
} 


\section{[Image 1. Foyer historique de la Gaîté lyrique @ Philippe Ruault]}

On se propose ainsi d'étudier les salles parisiennes à travers leurs programmations réelles et leurs manières de faire entendre la musique, en mêlant traitements statistiques et ethnographie. Le recours à une Analyse des Correspondances Multiples (ACM), réalisée à partir des propriétés objectives des salles, porte au jour la structure de cet espace. La méthode exerce ainsi un « pouvoir de révélation $»^{21}$ et rend visible la répartition des genres musicaux, qui sous-tend, d'une certaine manière, les «prises de position » artistiques des salles. Une ethnographie multisituée a permis de définir les variables pertinentes pour décrire les salles et d'interpréter les résultats statistiques ${ }^{22}$.

\section{Encadré 1 : L’analyse des correspondances multiples}

\section{Une reconnaissance institutionnelle inégale}

Si la légitimité culturelle a souvent été appréhendée par le prisme de la stratification sociale des goûts musicaux, l'analyse des offres de sortie permet de l'envisager au travers de sa dimension institutionnelle et de confronter les différentes manières d'entendre la musique qui sont proposées.

L'ACM montre que les salles de musique parisiennes se différencient d'abord par leur mode de financement et leur statut juridique. D'un côté se situent des salles bénéficiant de subventions publiques et des dons de mécènes. Il peut s'agir d'établissements publics (EP), voire

\footnotetext{
${ }^{21}$ Julien Duval, «L'analyse des correspondances et la construction des champs », Actes de la recherche en sciences sociales, $\mathrm{n}^{\circ} 200,2013$, p. 110-123.

${ }^{22}$ L'enquête a été conduite entre 2011 et 2014 en différents lieux de la capitale. Elle s'est accompagnée de la réalisation d'une trentaine d'entretiens avec des programmateurs de salles parisiennes et des représentants d'associations professionnelles ou de la Mairie, permettant de comprendre plus finement les principes de structuration de cet espace. Dans les salles elles-mêmes, les renseignements sur la station debout ou assis, les paliers de jauges, la présence d'un restaurant, les heures d'ouverture, l'histoire particulière du lieu etc., découlent de nos observations et recherches dans les documentations des salles.
} 
d'associations loi 1901. A l'autre pôle de l'espace se trouvent les entreprises privées au statut de SARL, de SAS, ou de SA, parmi lesquelles certaines proposent des prix d'entrée modiques (0 à $10 €)$. En plus de l'intégration de logiques, de pratiques et de réseaux spécifiques, la captation de financements extérieurs nécessite un certain degré de reconnaissance, notamment musicale, pour le lieu de diffusion. Le type de programmation de la salle importe donc, puisque celles qui proposent des activités destinées aux amateurs, ainsi que plus de 5 genres musicaux et une forte pluridisciplinarité (à gauche), s'opposent à des salles où n'est jouée que de la musique (à droite).

La présence importante sur les réseaux sociaux (plus de 30000 «like » sur Facbeook) des lieux caractérisés par le mécénat et les subventions publiques indique une visibilité forte parmi les publics, mais suggère également la valeur distinctive attribuée à leur fréquentation. Quant aux salles les plus éloignées de ce pôle institutionnel, ce sont aussi celles où la musique n'apparaîtrait pas comme première (le bar), et où la pratique musicale serait insuffisamment « désintéressée ${ }^{23}$, la boîte de nuit étant perçue avant tout à travers sa dimension d'entreprise économique et non artistique ${ }^{24}$. Deux modèles de sortie musicale se font donc face : d'une part les lieux de «concert », où le public se rend afin d'écouter la musique ; d'autre part, la boîte de nuit intégrant la danse, la « fête », la « nuit », et le bar musical, qui semblent plus fortement liés à des sociabilités. Notons que l'ouverture la nuit et le jour contribue sensiblement à structurer cet axe, avec à gauche des lieux qui ferment au plus tard à 2 heures et qui sont ouverts le jour, à droite ceux qui s'animent jusqu'à 5 heures du matin et ferment en journée. Philippe Coulangeon souligne que « la légitimité culturelle ne se définit pas seulement par rapport à la segmentation des genres mais encore par rapport à la différenciation des usages, en opposant

\footnotetext{
23 Sur l'éthique du désintéressement en lien avec la vocation artistique, voir notamment Gisèle Sapiro, « La vocation artistique entre don et don de soi », Actes de la recherche en sciences sociales, $\mathrm{n}^{\circ} 168,2007, \mathrm{p} .4-11$.

${ }^{24}$ Ainsi, un cadre de la Mairie de Paris explique « Maintenant, les boîtes de nuit à Paris, j'ai pas le sentiment que ça marche mal... Et je vois pas quelles missions de service public, artistique véritablement, elles adressent, donc ça me paraît pas pertinent qu'on vienne sur ce segment-là. », entretien réalisé le 21.05.2014.
} 
écoute "pure" et écoute "fonctionnelle" ${ }^{25}$. C'est bien cette opposition qui trouve à s'institutionnaliser dans les caractéristiques des salles, les publics étant toutefois susceptibles de circuler entre elles.

Cette distinction se manifeste également au niveau des genres musicaux. Les modalités supplémentaires (graphique 3) concernant la présence de musiques classiques, puis dans une moindre mesure de musiques expérimentales et du monde, s'opposent à la techno (à droite). Les modalités du rock, des variétés, de la pop, des musiques électroniques etc. occupent quant à elles des positions intermédiaires.

Tableau 1. Contributions (en \%) des modalités retenues à la variance de l'axe 1

\begin{tabular}{|c|c|c|c|c|}
\hline \multicolumn{5}{|c|}{ Axe 1. Reconnaissance institutionnelle $(7,12 \%$ de la variance du nuage) } \\
\hline \multirow{2}{*}{ Variables } & Modalité & Contribution & Modalité & Contribution \\
\hline & \multicolumn{2}{|c|}{ A droite } & \multicolumn{2}{|c|}{ A gauche } \\
\hline Subventions publiques & fin_public_non & 4,3 & financement_public & 12,08 \\
\hline \multirow{2}{*}{ Statut juridique } & & & Etab. Public & 10,19 \\
\hline & SARL & 1,58 & Association & 2,16 \\
\hline Pluridisciplinarité & 0_pluri & 3,79 & FortPluri_3_4 & 7,27 \\
\hline Mécénat & mécénat_non & 2,88 & mécénat_oui & 5,05 \\
\hline Ouverture la nuit & $5 \mathrm{H}$ & 2,98 & $2 \mathrm{H}$ & 1,72 \\
\hline Pratiques amateur & & & amateurs & 4,37 \\
\hline Ouverture en journée & fermé_jour & 1,55 & ouvert_jour & 2,21 \\
\hline Date d'ouverture & 2011 et après & 1,43 & 1990-1995 & 2,18 \\
\hline Page Facebook & & & $\begin{array}{l}\text { Facebook (plus de } \\
30000 \text { ) }\end{array}$ & 3,55 \\
\hline Localisation & & & Arr19 & 3,12 \\
\hline Jauge & & & Jauge $>1500$ & 2,53 \\
\hline Prix d'entrée & 0 à $10 €$ & 2,24 & & \\
\hline $\begin{array}{l}\text { Nombre de genres } \\
\text { musicaux }\end{array}$ & & & 5 genres ou plus & 1,65 \\
\hline Compte Twitter & Twitter_non & 1,52 & & \\
\hline
\end{tabular}

Les musiques classiques, «savantes » ou légitimes, sont depuis longtemps intégrées par la sphère institutionnelle et subventionnées. Quant aux musiques généralement qualifiées

\footnotetext{
${ }^{25}$ Philippe Coulangeon, « "La stratification sociale des goûts musicaux." Le modèle de la légitimité culturelle en question », art. cit., p. 15.
} 
d'« actuelles » par les politiques culturelles, Philippe Teillet analyse la difficulté pour leurs acteurs de s'insérer dans le champ des politiques culturelles et de légitimer leurs demandes selon les logiques qui l'organisent (un discours oscillant entre justifications sociales et intérêt artistique $)^{26}$. Il observe ainsi que les individus issus du monde du rock (notamment par leurs propriétés sociales et trajectoires) sont souvent plus avancés dans ce processus que ceux du rap ou de la techno.

Dans le cas parisien, l'ACM semble montrer que le rap, quoique très minoritaire (joué dans 17 salles), est relativement intégré aux salles du secteur subventionné. Cela peut s'expliquer par le fait qu'il n'existe aucune salle qui ne joue que du $\operatorname{rap}^{27}$, de même qu'il n'existe aucune salle qui présente uniquement de la noise ou du punk à Paris, ces genres figurant généralement parmi une programmation relativement variée relevant desdites «musiques actuelles ». Quoique la techno ne dispose pas non plus de salle spécialisée, elle est presque toujours jouée en lien avec d'autres musiques électroniques.

Quoique les situations des salles moins reconnues institutionnellement soient plutôt hétérogènes, les parcours des individus qui composent ces univers permettent certainement d'expliquer en partie cette distance. Certains d'entre eux, tels que les gestionnaires de Savoir Faire, agence qui exploite à la fois le Showcase (avec l'agence We are Paris) (75008), le Silencio (75002), le Social Club jusqu'à fin 2014 (75002) et le Wanderlust (75013) (sur le graphique 5), ainsi que différents festivals parisiens, ont privilégié l'intégration dans un marché de la musique en lien par exemple avec la publicité de grandes entreprises. D'autres appartiennent plutôt à la catégorie des débits de boisson qui ouvrent une arrière-salle ou débarrassent les chaises pour constituer une piste de danse, afin d'accueillir des DJs le weekend.

\footnotetext{
${ }^{26}$ Philippe Teillet, «Eléments pour une histoire des politiques publiques en faveur des "musiques amplifiées », in Philippe Poirrier, Les collectivités locales et la culture. Les formes de l'institutionnalisation, XIXè et XXè siècles, Paris, Comité d'Histoire de Ministère de la Culture, La Documentation Française, 2002, p. 361-393.

${ }^{27}$ Mais il importe également de mettre cela en regard de la politique ambivalente menée par les pouvoirs publics à l'égard de ce genre. Karim Hammou, Une histoire du rap en France, op. cit, p. 119-141.
} 


\section{La renommée, principe structurant de l'espace des salles}

Le modèle économique et artistique n'est pas l'unique principe de différenciation des salles.

Elles se distribuent également en fonction de leur plus ou moins grande renommée sur les plans régional, national, voire international (axe 2), qui pourrait également se traduire en termes de volume global de capital symbolique. Les salles les plus renommées sont les plus susceptibles d'attirer un public de touristes. Parmi les modalités contribuant le plus fortement à la structuration du second axe, la jauge, c'est-à-dire le nombre de spectateurs que la salle peut accueillir, figure comme une caractéristique centrale (voir tableau 2).

Grandes et petites salles se distinguent nettement sur cet axe. Sous ce rapport, la visibilité numérique (page Facebook très suivie) et le prix des places sont clivants. Cela souligne également la valeur sociale attribuée à la salle et à sa programmation par les publics, et en retour, la distinction que peut conférer la revendication publique de leur connaissance ou fréquentation. De même, la localisation géographique oppose rive droite («8 et $16^{\text {ème }}$ arrondissement $»$ et $« 2^{\text {ème }}$ arr. ») et rive gauche (« 5,6 et $15^{\text {ème }}$ arr. $\left.»\right)$, ainsi que ouest et est parisien («20 $0^{\text {ème }}$ arr. ») Du côté des salles les moins renommées, l'offre de pratiques artistiques pour les amateurs témoigne de la moindre séparation entre profanes et professionnels qui existe dans ces lieux. De plus, l’impossibilité de les privatiser et leur statut juridique («Autre », donc commerçants etc.) soulignent la moindre spécialisation de certains de ces espaces dans l'activité de spectacle ou l'offre festive, ce que l'opposition entre l'ouverture en journée ou seulement en soirée traduit également.

Tableau 2. Contributions (en \%) des modalités retenues à la variance de l'axe 2

\begin{tabular}{|c|c|c|c|c|}
\hline \multirow{2}{*}{ Variables } & Modalité & Contribution & Modalité & Contribution \\
\hline & \multicolumn{2}{|c|}{ En haut } & \multicolumn{2}{|c|}{ En bas } \\
\hline \multirow{2}{*}{ Jauge } & & & $\begin{array}{l}100 \text { places ou } \\
\text { moins }\end{array}$ & 8,82 \\
\hline & $\begin{array}{l}1001 \text { à } 1500 \\
\text { places }\end{array}$ & 3,22 & & \\
\hline
\end{tabular}




\begin{tabular}{|c|c|c|c|c|}
\hline Page Facebook & \begin{tabular}{|l}
10001 à \\
30000 \\
«like » \\
Plus de \\
30000 \\
« like »
\end{tabular} & 5,07 & Sans_Facebook & 6,08 \\
\hline \multirow{2}{*}{ Arrondissement } & Arr8et16 & 3,89 & Arr5et6et15 & 2,31 \\
\hline & Arr2 & 1,92 & Arr20 & 2,15 \\
\hline Ouverture en journée & fermé_jour & 2,75 & ouvert_jour & 3,93 \\
\hline Prix d'entrée & 51 à $100 €$ & 2,53 & 0 à $10 €$ & 4,12 \\
\hline Statut juridique & SAS et SA & 3,81 & Autre & 2,62 \\
\hline Pratiques amateur & & & amateurs & 5,49 \\
\hline Compte Twitter & Twitter_Oui & 1,81 & Twitter_Non & 2,1 \\
\hline Date d'ouverture & & & $1996-2000$ & 2,87 \\
\hline Pluridisciplinarité & & & FortPluri_3_4 & 2,17 \\
\hline $\begin{array}{l}\text { Privatisation de la } \\
\text { salle }\end{array}$ & & & privatisation_non & 2,08 \\
\hline Ouverture la nuit & $5 \mathrm{H}$ & 1,98 & & \\
\hline
\end{tabular}

Du côté des petites salles, se trouvent les modalités indiquant une programmation de jazz et de musiques expérimentales (graphique 5), puis de manière moins marquée de rap et de musiques du monde. De l'autre côté, se situent l'opéra et opérette, les variétés, techno, pop et musique contemporaine. Cet axe oppose également la «programmation très peu internationalisée » $(0$ à 10\%) aux autres modalités de cette variable.

\section{[Image 2. Salle Favart de l'Opéra Comique (C) Christophe Chavan]}

Au pôle dominant sur le plan de la renommée se dessine une géographie des salles relativement hétérogène (en haut sur le graphique 5) : artistes programmés attirant un public important en nombre, notamment dans les variétés ${ }^{28}$ (Bataclan, Grand Rex, Olympia...), prix des places élevés, dans les maisons d'opéra et de musiques classiques (Théâtre des Champs Elysées, Pleyel - au contraire de la Cité de la Musique) ou dans les boîtes de nuit de luxe du $8^{\text {ème }}$

\footnotetext{
${ }^{28}$ Rappelons toutefois que les esthétiques et les artistes recouverts par ce terme sont très variables et qu'il s'agit d'une catégorie dont le temps et les (in)succès contribuent également à redéfinir les contours.
} 
arrondissement, dont l'entrée est sélective, la table réservable avec bouteille d'alcool fort à la clef et un valet de parking disponible (79 Club, Queen, ZigZag).

La sociologie des pratiques culturelles insiste fréquemment sur la fracture entre musiques « savantes » et «populaires »: les propriétés sociales de leurs publics et de leurs artistes se distingueraient fortement et la légitimité qui leur est associée les séparerait radicalement. Cependant, les très grosses salles de ces deux catégories, dont la programmation fortement internationalisée compte souvent des vedettes ${ }^{29}$, sont comparables sur le plan de la renommée, même si celle-ci ne se constitue pas nécessairement auprès des mêmes publics.

L'exemple de la salle Pleyel est ici révélateur, car il semble bien illustrer ce phénomène : la nouvelle Philharmonie, qui compte 2400 places, ouvrant dans le $19^{\text {ème }}$ arrondissement (2015), la salle Pleyel ne sera plus exploitée par la filiale de la Cité de la Musique. Selon un cadre de la Mairie de Paris chargé des projets en lien avec la musique, afin d'éviter une trop forte concurrence de l'offre de musiques classiques situées dans l'ouest parisien, l'appel d'offre pour son exploitation exclut ces dernières de sa programmation future. Il suppose qu' « on va plutôt s'acheminer vers une forme d'Olympia bis, enfin un lieu qui fera de la variété plus plus plus $»^{30}$. Les caractéristiques de la salle lui permettraient donc cette versatilité. Le directeur d'une salle prestigieuse de musiques classiques explique quant à lui que les variétés constituent «peut-être 5\% » de la programmation. Il précise : «En général, tout ce qui est variétés, c'est fait avec des producteurs extérieurs. [...] Je fais quand même intervenir mes goûts personnels, c'est-à-dire [...] si on me fait une demande pour Bénabar qui veut faire un truc acoustique, je dirai oui, parce que ça me semble correspondre plus à l'image que je souhaite donner du théâtre » ${ }^{31}$. Dans sa programmation, le Théâtre du Châtelet inclut également des «variétés », au sens large (musicals, humour et stand-up) défendu par le Centre National des Variétés, dans

\footnotetext{
${ }^{29}$ La programmation de «stars » ne touche toutefois pas également ni de la même manière musiques classiques et variétés.

${ }^{30}$ Entretien réalisé avec un cadre de l'administration de la Mairie de Paris, le 21.05.2014.

${ }^{31}$ Entretien réalisé avec le directeur d'une salle de musiques classiques à grande jauge, le 26.09.2014.
} 
une optique de diversification des publics, mais aussi de rentabilité financière : « [à propos de la programmation de l'humoriste Florence Foresti] c'était l'occasion d'ouvrir en grand les portes du théâtre et de faire en sorte que le "modèle" du Châtelet (une programmation éclectique, populaire et sophistiquée) ne soit pas toujours le même. [...] Il y a encore un sacré boulot à faire pour élargir le public. Ma conviction est qu'une fois qu'on a osé y entrer, on peut y retourner. $»^{32}$ Le Grand Rex présente différents orchestres symphoniques, l'un d'entre eux étant dirigé par le compositeur Vladimir Cosma (avec certains solistes issus des variétés), qui a précédemment programmé aux théâtres du Châtelet et des Champs Elysées. A l'échelle de la capitale, les caractéristiques de la sortie (taille de la salle, prix des places etc.) reconfigurent peut-être différemment l'opposition entre les variétés et les musiques classiques, quoique leur co-programmation dans un même lieu demeure marginale (5 salles programment les deux sur les 41 diffusant l'un et/ou l'autre des deux genres).

La position similaire des maisons d'opéra, salles de musiques classiques et de certaines boîtes de nuit (Showcase, ZigZag, Yoyo etc.) pose également question. On peut induire du prix des places et de l'observation des publics (notamment des choix vestimentaires) que les fractions de la population bien dotées en capital économique sont très représentées parmi ceux qui les fréquentent, la différence principale tenant à l'âge. Selon la personne s'occupant de la communication d'une autre boîte de nuit ${ }^{33}$, les publics du Club 79 aux Champs Elysées, sont mélangés. Selon lui, ils n'appartiennent pas à la bourgeoisie traditionnelle, contrairement à ceux du Yoyo, situé dans le Palais de Tokyo, qu'il qualifie de «très $16^{\text {ème }} 8^{\text {ème }}$ ». Cependant, si certains de ceux qui fréquentent le Club 79 sont avant tout des amateurs de hip-hop, d'autres disposent bien d'un capital économique élevé qu'atteste la réservation de carrés VIP qui se

\footnotetext{
32 « Programmer Florence Foresti permet de casser les frontières », entretien de Jean-Luc Choplin avec Sandrine Blanchard, Le Monde, 7 octobre 2014, disponible en ligne http://www.lemonde.fr/scenes/article/2014/10/07/jeanluc-choplin-programmer-florence-foresti-permet-de-casser-les-frontieres_4501963_1654999.html, consulté le 5 janvier 2015.

${ }_{33}^{33}$ Entretien le 11.17.2014, avec un responsable de la communication d'une boîte de nuit appartenant au groupe Noctis, qui rassemble 17 lieux de nuit parisiens, duquel le Club 79 et le Yoyo font également partie.
} 
« vendent » à plusieurs milliers d'euros pour une soirée. De plus, la venue des publics les plus dotés en capital économique repose sur un fonctionnement en clique, où des «rabatteurs » informels font venir des connaissances, puis sont rémunérés grâce à un pourcentage sur les tables réservées. La Machine du Moulin Rouge et le Rex Club font quant à eux figure d'exception, car leurs publics sont plus diversifiés et leur approche du « club » différente. Néanmoins, l'apport en termes d'image sociale et les sociabilités engagées paraissent parfois rapprocher la sortie dans ces boîtes de nuit particulières de certains rituels sociaux du concert de musiques classiques, en dépit de différences marquées quant à la mise en scène du corps, notamment pour les femmes. Un parallèle peut ainsi être dressé entre certaines manières de rendre visible son statut social et de sortir, comme la réservation d'une table ou de sa place au parterre, l'exposition de son passage dans ces lieux et de ses différents capitaux ${ }^{34}$. L'importance des ressources économiques est plus ou moins euphémisée en fonction des espaces et des fractions de classe qui y sont prédominantes. Bien entendu, ces lieux attirent également des individus souhaitant s'intégrer à des groupes sociaux dont ils ne font pas (encore) partie, mais l'entre-soi y est renforcé par une sélection économique et culturelle de la clientèle, ainsi que par le «physionomiste » à l'entrée. De la même manière que Christophe Charle établit une correspondance entre situation spatiale et position sociale à la fin du XIXème siècle, « les écrivains les plus lancés élis[a]nt domicile dans les quartiers de l'ouest parce qu'ils sont académiciens ou ont eu les succès les plus brillants $»^{35}$, il est significatif qu'une partie de ces établissements se situe dans un même espace géographique. Celui-ci se caractérise par de très

\footnotetext{
${ }^{34}$ Les photos des soirées précédentes mises en ligne, plus souvent que pour d'autres salles, rendent visibles des personnes individualisées, posant généralement face caméra (principalement des femmes). Il s'agit rarement de photos prises par les publics. Quoique cette question mérite des approfondissements, il s'agit également d'une manière pour la salle de revendiquer le statut social des individus qui la fréquentent et leur attractivité physique, notamment celle des femmes. Sur ces questions, voir notamment Bertrand Réau, « Enchantements nocturnes : ethnographie de deux discothèques parisiennes », Ethnologie française, , n², 2006, p. 333-339.

${ }^{35}$ Christophe Charle, «"Situation spatiale et position sociale" Essai de géographie sociale du champ littéraire à la fin du XIXème siècle », art. cité, p. 50.
} 
hauts revenus ${ }^{36}$ ( $8^{\text {ème }}$ et $16^{\text {ème }}$ arr.) et une proximité avec les banlieues les plus favorisées, les critères d'accès à la salle conditionnant un recrutement géographique large de leurs publics, y compris auprès des touristes (la boîte le Queen les cible ainsi en particulier) ${ }^{37}$. La segmentation des styles de sortie et des modes de recrutement des publics de ces boîtes de nuit organise cependant leur répartition sociale entre les différents lieux.

L'axe 2 oppose à ces salles dominantes, dont la fonction tourne autour du « spectacle » (elles n'ouvrent qu'en soirée), que celui-ci soit musical ou social, de plus petites jauges, ouvertes en journée, qui entretiennent des sociabilités et une visibilité de jour. Cela peut se traduire par l'organisation d'expositions (Atelier du Plateau, Institut des Cultures d'Islam où il s'agit de l'activité principale), l'existence d'un café-bar (Café Universel, Ogresse, 3 Arts qui dispose également d'une AMAP), et/ou la possibilité d'accueillir des pratiques amateur (Centre Barbara Fleury Goutte d'Or, Manufacture Chanson). Leurs prix d'entrée sont généralement peu élevés, les artistes présentés étant moins connus, à l'exception de Barbara FGO et de l'Institut des Cultures d'Islam qui sont des lieux plus visibles.

Ici aussi, la répartition des genres musicaux dans l'espace dessiné (graphique 3) révèle des expériences musicales différentes. Le jazz et les musiques expérimentales se jouent généralement dans de petites salles (le jazz est présent dans $31 \%$ des salles, dont plus de 50\% ont une jauge inférieure à 201 places). Au contraire, $43 \%$ des salles présentant des variétés ont une jauge supérieure à 1000 places, le chiffre étant identique pour les musiques classiques, diffusées dans seulement $15 \%$ des salles parisiennes. Les salles diffusant du jazz, proches du pôle moins visible de l'axe 2, semblent relever de ce que Wenceslas Lizé identifie comme le « jazz traditionnel $»^{38}$, fortement lié au dispositif du « club». Si la majorité d'entre elles ne

\footnotetext{
${ }^{36}$ En 2009, le revenu médian par ménage dans le $8^{\text {ème }}$ arrondissement est de $49060 €$ et de $46151,5 €$ dans le $16^{\text {ème }}$, les plus hauts de la capitale. Source des données : Direction Générale des Impôts, traitement Insee.

${ }^{37}$ De manière similaire à la société théâtrale du West End, dont le modèle économique est fondé sur le luxe quoiqu'elle demeure hétérogène, analysée dans Christophe Charle, Théâtres en capitales. Naissance de la société du spectacle à Paris, Berlin, Londres et Vienne, op. cit., en particulier p. 258-264.

${ }^{38}$ Wenceslas Lizé, «"Le goût jazzistique en son champ" L'espace parisien de la jazzophilie », art. cit.
} 
correspond pourtant pas exactement à l'idéaltype du «club», tendant plutôt vers le barrestaurant, les mises en scène du lieu (dans la salle ou dans leur communication) mobilisent bien cette mythologie. Ces petites salles sont souvent bien connues des habitués et auprès de certains cercles spécifiques, mais moins identifiées dans l'espace musical général.

Le plan formé par les axes 1 et 2 permet de découper l'ensemble des salles en quatre groupes (graphique 5). En bas à gauche, les salles de plus petites jauges, privilégiant notamment les pratiques amateurs et la pluridisciplinarité, bénéficiant d'une reconnaissance institutionnelle (financements publics et mécénat), parmi lesquelles se situent le jazz et les musiques expérimentales, que le Lavoir Moderne Parisien illustre bien. Il dispose d'une petite salle en gradins, séparée du bar, où l'écoute alterne entre manifestation festive et concentration recueillie en fonction des propositions, plus ou moins improvisées ou expérimentales. Opposées à elles sur le plan de la reconnaissance institutionnelle (en bas à droite), les salles dont l'activité principale (APE) relève plutôt du débit de boissons ou de la restauration traditionnelle, également peu visibles sur internet, aux prix plutôt bas et avec une programmation peu internationalisée et peu connue. Par exemple, le Café des Sports fait l'angle d'une rue près de Ménilmontant et «contribue à cette vie de quartier en proposant une offre musicale hétéroclite ${ }^{39}$ dans un cadre dont la disposition est celle du café, avec comptoir en zinc, sans réelle scène ni dispositif technique développé.

Dans le cadre en haut à droite, les salles privées, plus internationalisées et connues, se rapprochant du dispositif «boîte de nuit», fermées le jour, ouvertes tard dans la nuit, rassemblent les genres musicaux house et techno. Et s'opposant à elles, le dernier quart (en haut à gauche), des lieux connus et institutionnellement reconnus, visibles, plus chers, présentant plutôt des musiques classiques et variétés, un certain nombre d'entre eux étant construits sur un modèle semblable à la salle de théâtre. Ainsi la Cigale, théâtre à l'italienne avec balcon et

\footnotetext{
39 «Le lieu », http://lecafedessportsmenilmontant.com/le-lieu/, consulté le 25.05.2014.
} 
coloris rouge sombre, présente principalement des «musiques actuelles », mais adapte les configurations assises et/ou debout au type de musique en concert.

\section{L'ancienneté des salles et l'accumulation de capital symbolique}

Le troisième axe révèle un principe de polarisation différent. Il s'agit de l'ancienneté différentielle des salles (graphique 2). En fonction de celle-ci, non seulement les salles ne sont pas occupées de la même manière (on y est soit assis, soit debout), mais elles occupent des emplacements différents dans Paris : des arrondissements où les salles n'ont ouvert que plus récemment $\left(13^{\text {ème }}\right)$ s'opposent ainsi à ceux plus anciennement colonisés par la musique $\left(9^{\text {ème }}\right.$, $\left.2^{\text {ème }}, 4^{\text {ème }}, 5^{\text {ème }}\right)$. Des jauges moyennes font également face aux très grosses. Les salles de 201 à 300 places $(13 \%$ de l'ensemble), sont relativement récentes $(60 \%$ ont été créées à partir des années 2000), et plus de $80 \%$ des jauges de 301 à 600 places ont été ouvertes à partir des années 1990. A cette époque, les politiques publiques envers les musiques dites actuelles se renforcent, notamment avec la création en 1989 d'une Agence nationale pour le développement des petites salles de spectacles, qui concerne celles de 80 à 800 places.

A l'opposé, deux tiers des jauges supérieures à 1500 places ont ouvert avant les années 1990, dont trois dans le $9^{\text {ème }}$ arrondissement. Ces salles plus anciennes (avant les années 1990), dont les entrées sont plus chères et qui ferment généralement avant $2 \mathrm{~h}$, sont les plus représentées (près de 50\%) parmi les lieux affichant un genre musical unique. Les plus récentes, dont les places sont moins onéreuses (11 à 20€) et qui peuvent être ouvertes plus tard la nuit, ont une programmation musicale fortement diversifiée, et plus souvent très pluridisciplinaire. L'association entre les heures de fermeture et le type de placement des publics relève des expériences et manières d'entendre la musique différentes, qui s'échelonnent à la fois entre les genres musicaux, mais aussi entre des publics dont l'âge et l'appartenance générationnelle divergent. 
Tableau 3. Contributions (en \%) des modalités retenues à la variance de l'axe 3

\begin{tabular}{|c|c|c|c|c|}
\hline \multicolumn{5}{|c|}{ Axe 3. Temps social $(5,44 \%$ de la variance du nuage) } \\
\hline \multirow{2}{*}{ Variables } & Modalité & Contribution & Modalité & Contribution \\
\hline & \multicolumn{2}{|c|}{ A droite } & \multicolumn{2}{|c|}{ A gauche } \\
\hline Position du public & Debout & 6,14 & Assis & 10,04 \\
\hline \multirow{3}{*}{ Arrondissement } & Arr13et14 & 3,38 & Arr9 & 3,87 \\
\hline & & & Arr4et12 & 1,46 \\
\hline & & & Arr5et6et15 & 1,76 \\
\hline \multirow{3}{*}{ Date d'ouverture } & & & Avant 1950 & 6,2 \\
\hline & & & 1981-1989 & 1,63 \\
\hline & & & $1950-1980$ & 1,45 \\
\hline \multirow{2}{*}{ Prix d'entrée } & & & $>100 €$ & 4,75 \\
\hline & 11 à $20 €$ & 2,26 & 51 à $100 €$ & 2,11 \\
\hline Jauge & $\begin{array}{l}201 \text { à } 300 \\
\text { places }\end{array}$ & 3,78 & Jauge $>1500$ & 4,98 \\
\hline $\begin{array}{l}\text { Nombre de genres } \\
\text { musicaux }\end{array}$ & 5 genres ou + & 1,84 & 1 genre & 5,65 \\
\hline Horaires de fermeture & $5 \mathrm{H}$ & 4,12 & $2 \mathrm{H}$ & 2,38 \\
\hline Pluridisciplinarité & FortPluri_3_4 & 3,92 & MoyenPluri_1_2 & 2,11 \\
\hline Compte Twitter & Twitter_Oui & 1,56 & Twitter_Non & 1,8 \\
\hline Pratiques amateur & amateurs & 1,9 & & \\
\hline
\end{tabular}

Si l'on regarde la répartition des genres en variables supplémentaires, c'est du côté des plus anciennes salles, à gauche de l'axe, qu'apparaissent les musiques classiques (en particulier l'opéra et l'opérette) et variétés (graphique 4). Le jazz et la chanson se situent près du centre. A droite, se trouvent tous les autres genres musicaux, avec de manière plus marquée la techno, les musiques expérimentales et électroniques.

La gradation du temps sur l'axe 3 correspond à un processus pérennisation de certaines salles et d'une certaine manière, des genres musicaux qu'elles présentent, semblable à celui décrit par Pierre Bourdieu à propos du champ littéraire : «Les différences qui séparent les petites entreprises d'avant-garde des "grosses entreprises" et des "grandes maisons" se superposent à celles que l'on peut faire, du côté des produits, entre le "nouveau", provisoirement dépourvu de valeur "économique", le "vieux", définitivement dévalué, et l'"ancien" ou le "classique", doté 
d'une valeur "économique" constante ou constamment croissante $»^{40}$. Le plan formé par les axes 2 et 3 illustre la manière dont cette pérennisation peut aller de pair avec l'accumulation de capital symbolique, que le temps contribue à renforcer et à naturaliser. La répartition des modalités de prix d'entrée semble aller dans le sens de cette valeur économique attribuée aux produits, en particulier pour les «classiques ». Cela ne doit pas éclipser le fait que certaines salles de création récente sont vite devenues des entreprises rentables économiquement, notamment par la magie du regroupement d'activités (être à la fois producteur, tourneur, diffuseur etc.) et l'exploitation de plusieurs lieux dans la capitale. C'est toutefois le cas d'une minorité de salles se situant plutôt au pôle renommé de l'axe 2. D'autres salles au pôle plus ancien sont quant à elles touchées par un phénomène de vieillissement social et de dévaluation de l'offre artistique présentée. Toutefois, cela n'exclut jamais un renversement et un processus de « classicisation », notamment à travers le réinvestissement des lieux (et des quartiers) par de nouveaux publics aux propriétés sociales différentes.

\section{Encadré 2. Les discours de présentation des lieux}

Le croisement des axes 2 (renommée) et 3 permet ainsi d'entrevoir la formation de trois groupes principaux (graphique 6). D’une part (en bas à gauche) se trouvent des petites salles créées pour la plupart dans les années 1990, plutôt proches du modèle «café-concert » et du jazz ou de la chanson. En glissant le long de l'axe 2 vers le haut, un groupe de cabarets fait le lien avec des salles généralement plus proches du dispositif théâtral, avant de passer aux salles de musiques classiques traditionnelles (concert et opéra), caractérisées par un important capital symbolique, à la fois en termes d'ancienneté et de renommée. En se déplaçant vers le côté droit, donc vers des salles plus récentes, les premières qui apparaissent sont principalement des salles de variétés

\footnotetext{
${ }^{40}$ Pierre Bourdieu, Les Règles de l'art, Paris, éditions du Seuil, 1998, p. 249.
} 
et des boîtes de nuit luxueuses, proches de la modalité « $8^{\text {ème }}$ et $16^{\text {ème }}$ arr. », précédemment évoquées. En descendant du cadran droit en haut à celui en bas, se déploie un continuum passant des boîtes de nuit et salles de concert plus traditionnelles, à des lieux plus mixtes et moins visibles. Parmi ces derniers, se rencontrent des espaces aux programmations plus confidentielles et éclectiques, souvent reconnus par la critique et par les administrateurs de politiques culturelles pour la qualité artistique de leur programmation (le Point Ephémère par exemple). Leurs jauges sont également plus restreintes. Ainsi, les spécificités des salles différencient plusieurs groupes emblématiques de la forme que peuvent prendre les lieux de musique, tout en rendant visible la gradation des multiples espaces moins identifiables et moins connus, aux caractéristiques plus mixtes, qui peuplent les interstices de la ville.

Les programmateurs comparent d'abord leur lieu aux salles les plus semblables (notamment en termes de jauges et de genres diffusés). Cependant, l'approche relationnelle permet de souligner différents principes de structuration de l'espace parisien des salles de musique, qui s'analysent à différentes échelles de rayonnement géographique. Se dessine ainsi l'opposition entre salles et pratiques de sortie bénéficiant d'une reconnaissance institutionnelle inégale, mais aussi entre salles dominantes du point de vue de la renommée et salles moins visibles. L’ACM dévoile également, on l' a vu, le poids de l'ancienneté des salles. Ces trois points semblent concomitants de la stabilisation de certains genres musicaux dans cet espace musical.

La sortie musicale engage des phénomènes différents de l'écoute par d'autres supports. La réception y est plus fortement cadrée par le dispositif d'appréciation que constitue la salle, par ses caractéristiques objectives et l'utilisation qui en est faite par les artistes et les intermédiaires, notamment les programmateurs. Les offres s'articulent à des manières de sortir diverses, qui peuvent s'opposer ou être associées par les publics : aller au concert ou au spectacle, rentrer tard chez soi, visiter un lieu pluridisciplinaire, payer sa place à la billetterie ou s'installer au 
comptoir, danser ou s'asseoir, boire, manger, écouter dans le noir, se montrer, venir voir un artiste très connu ou des amis qui jouent etc. Les genres musicaux se répartissent entre les lieux et pratiques, certains à cheval sur différents espaces sociaux, d'autres plus marqués par un type de salle et une manière d'entendre la musique.

\section{[Image. Radio Minus Soundsystem à la Gaîté lyrique @ Luca Lomazzi]}

La prise en compte simultanée de genres musicaux habituellement opposés par la sociologie des pratiques culturelles, et de lieux distincts de la salle de concert traditionnelle, rend visible les relations particulières qui se configurent à l'échelle de la capitale, tel que le positionnement proche sur le plan de la renommée et du prix des places des variétés et musiques classiques. Cela permet d'examiner le poids relatif donné à chacun et l'espace des possibles qui leur est offert en termes de programmations et de salles. Les mêmes genres musicaux ne sont pas investis des mêmes significations en fonction des espaces géographiques et sociaux dans lesquels ils sont ancrés. Ils sont vécus et appréciés très différemment selon la nature des lieux dans lesquels ils sont présentés, qui leur offriront une résonnance à des échelles variées : locales, nationales ou internationales. Dans une ville où un très grand nombre de salles mêle plusieurs genres musicaux, cela nous permet également de nourrir la réflexion sur la notion d'éclectisme.

Si la focale de l'éclectisme ne devrait sans doute pas être déplacée de l'écoute de différents genres musicaux aux pratiques de sortie, elle pourrait toutefois être mise en lien avec ces dernières. Quoique certains groupes d'individus fréquentent des lieux proposant diverses manières d'entendre la musique, l'inégale reconnaissance institutionnelle des salles semble constituer un indicateur de la perpétuation d'échelles de légitimité entre ces pratiques. Le soutien de la Ville de Paris pour des salles qui adaptent les modalités d'écoute des genres, en 
présentant par exemple des musiques électroniques dans des formats plus proches des musiques dites savantes, ou encore des musiques classiques avec un public debout, une bière à la main (comme à la Gaîté lyrique), semble également participer d'une légitimation et d'une valeur sociale conférées par les manières d'entendre la musique. Cela paraît marquer aussi les stratégies institutionnelles de positionnement de la ville parmi les capitales européennes et internationales, concomitantes d'un discours construisant la «nuit festive » comme capital symbolique, valorisable économiquement, à l'international. La distinction qu'apportent les différentes pratiques semble toujours structurer l'espace des salles et pouvoir démarquer certains publics. Ainsi, à rebours des représentations de la « nuit » et du concert, qui voudraient que se crée un instant de carnaval, espace fluide sans frontière où les identités sociales sont remisées au placard, possibilité de toutes les rencontres et de toutes les déviances interdites en journée, il semblerait plutôt que ces fantasmes de communion se segmentent en différents lieux aux positions et aux légitimités identifiables et qui demeurent inégales. 


\section{Encadré 1 : L'analyse des correspondances multiples}

L'ACM porte sur une base de données que nous avons construite, recensant les différentes salles de Paris (au nombre ici de 153). Elle s'appuie sur 18 variables actives (renvoyant à 69 modalités actives), les modalités «non-réponses » (NR), indiquées en italiques, ayant été mises en modalités passives. Dix-sept variables ont été retenues en tant qu'éléments supplémentaires $^{41}$.

\section{Les caractéristiques des salles}

\section{Spécificités du lieu physique}

L'expérience musicale faite par le public, au-delà de la proposition artistique, est également cadrée par le dispositif matériel dans lequel elle a lieu. Ces variables, les deux premières en particulier, rentrent également en compte dans la manière dont les programmateurs orientent leur sélection d'artistes.

- $\quad$ Jauge de la salle (7 modalités actives). Nous avons choisi ici de distinguer les très petites salles des petites salles, étant donné que la moitié des salles de Paris fait 300 places ou moins : 100 places ou moins $(n=24), 101$ à $200(n=31), 201$ à $300(n=20), 301$ à 600 $(\mathrm{n}=36), 601$ à $1000(\mathrm{n}=11), 1001$ à $1500(\mathrm{n}=12)$, plus de $1500(\mathrm{n}=12), N R(n=7)$.

- $\quad$ Type de placement ( 3 modalités actives) : pas de places assises (Debout ; n=36), places toutes assises (Assis ; n=57), mélange d'espaces où l'on est debout et assis (D/A ; n=60). Les espaces où il n'existe qu'un bar avec un comptoir agrémenté de quelques sièges hauts ont été classifiés comme n'ayant pas de places assises.

\footnotetext{
${ }^{41}$ Une variante de l'ACM, dite «ACM spécifique », a été utilisée. Elle évite que les modalités non pertinentes contribuent à la formation des axes. Voir Brigitte Le Roux et Henry Rouanet, Geometric Data Analysis. From Correspondence Analysis to Structured Data Analysis, Dordrecht, Kluwer Academic Publishers, 2004 ; Brigitte Le Roux, « Analyse spécifique d'un nuage euclidien : application à l'étude des questionnaires », Mathématiques, informatique et sciences humaines, 146, printemps 1999, p. 65-83; Id, Analyse géométrique des données multidimensionnelles, Paris, Dunod, 2004, en particulier le chapitre 8 et les pages 378 à 394 .
} 
- Présence d'un restaurant (2 modalités actives) : Restaurant_oui ( $\mathrm{n}=72)$, Restaurant_non $(n=81)$.

\section{Capital symbolique}

A l'instar de Pierre Bourdieu dans son étude des maisons d'édition, nous avons choisi de ranger la localisation et l'ancienneté dans les variables indiquant un type de capital symbolique. Les représentations sociales associées aux différents arrondissements, les différentes populations qui les habitent et les fréquentent, puis les équipements, culturels et autres, qui s'y répartissent indiquent qu'il est raisonnable d'estimer que situations spatiale et sociale sont souvent liées ${ }^{42}$. L'emplacement géographique joue effectivement un rôle dans la (non)captation et les bassins de recrutement de différents types de publics et dans les manières de se percevoir et de programmer.

- Localisation dans Paris (12 modalités actives) : certains arrondissements ont été regroupés afin d'éviter de trop petits effectifs. Nous avons ainsi réuni des arrondissements ayant des frontières communes, en regardant également comment ils se situaient individuellement en modalités illustratives sur les axes 1, 2 et 3 : Arr1et3 ( $n=12)$, Arr2 $(n=11), \operatorname{Arr} 4$ et12 (n=9), Arr5et6et15 ${ }^{43}(n=10)$, Arr6et16 $(n=13), \operatorname{Arr} 9(n=10)$, Arr10 $(n=12), \operatorname{Arr11}(n=23), \operatorname{Arr13et} 14(n=13), \operatorname{Arr17et18}(n=17), \operatorname{Arr19}(n=13), \operatorname{Arr} 20(n=10)$.

- $\quad$ Ancienneté de la salle (8 modalités actives) : les dates retenues sont celles de la création de l'organisme qui gère actuellement la salle. La direction artistique du lieu peut ainsi avoir changé depuis. Le découpage a privilégié des temps plus resserrés pour la période récente (depuis 1990), la création de salles encore en activité aujourd'hui s'y étant

\footnotetext{
${ }^{42}$ Voir par exemple Christophe Charle, «"Situation spatiale et position sociale" Essai de géographie sociale du champ littéraire à la fin du XIXème siècle », Actes de la recherche en sciences sociales, n 13, 1977, p. 45-59; Pierre Bourdieu, «Effets de lieu », in Pierre Bourdieu (dir.), La misère du monde, Paris, Le Seuil, 1993.

${ }^{43}$ Une seule salle se trouve dans le $15^{\text {ème }}$, à la frontière des $5^{\text {ème }}$ et $6^{\text {ème }}$ arrondissements, ce qui explique ce regroupement qui pourrait autrement paraître surprenant.
} 
accéléré. 1981 a également été retenu en raison de l'arrivée de Jack Lang au ministère de la Culture, qui a contribué à l'élargissement des domaines d'intervention publique en matière de culture ${ }^{44}$, y compris dans le domaine musical : Avant 1950 (n=13), 1950-1980 $(n=13), 1981-1989(n=15), 1990-1995 \quad(n=14), 1996-2000 \quad(n=20), 2001-2005(n=21)$, 2006-2010 $(n=30), 2011$ et après $(n=22)$.

- La dotation en subventions publiques (2 modalités actives), celles-ci étant regroupées quelle que soit l'institution qui les dispense (ministères, Ville de Paris, région Ile-deFrance etc.). Comme le mécénat, cette variable a été classée parmi les indicateurs de capital symbolique d'une salle, car elle restitue une forme de reconnaissance. Dans le cas des subventions publiques, celui-ci peut se faire au nom de logiques artistiques ou sociales. Dans le cas du mécénat, ce soutien peut également avoir trait à l'image que souhaite se donner l'entreprise ou l'individu : financement_public $\quad(n=40)$, fin_public_non $(\mathrm{n}=109), N R(n=4)$.

- Le mécénat de la salle par des partenaires privés (2 modalités actives) : mécénat_non ( $\mathrm{n}=85)$, mécénat_oui $(\mathrm{n}=48), N R(n=20)$.

- L'installation de la salle dans un lieu ayant une histoire particulière (en lien avec la musique ou par le biais du réinvestissement d'une « friche industrielle), valorisée dans la communication (2 modalités actives) : ancien_lieu ( $n=68)$, NR_lieu ( $n=85)$.

\section{Statut juridique et modèle économique}

- Statut juridique (5 modalités actives) : associations loi de 1901 (Association ; n=15), établissements publics (EP; $n=12)$, SARL ( $=69)$, Sociétés anonymes et sociétés par actions simplifiées (SAS et $\mathrm{SA} ; \mathrm{n}=36$ ), autres statuts, regroupant notamment les commerçants (Autre ; $\mathrm{n}=12), N R(n=9)$.

\footnotetext{
${ }^{44}$ Voir notamment Vincent Dubois, La Politique culturelle. Genèse d'une catégorie d'intervention publique, Paris, Belin, 1999.
} 
- Les prix des places : La variable prix constitue un critère décisif, qui oriente la sortie : il montre ce que chacun est en mesure de et prêt à payer en fonction de la programmation et du lieu. Dans les cas où les prix d'entrée sont variables, le prix le plus cher est retenu et non pas le prix moyen (6 modalités actives) : 0 à $10 €(n=35), 11$ à $20(n=47), 21$ à 30 $(\mathrm{n}=27), 31$ à $50(\mathrm{n}=12), 51$ à $100(\mathrm{n}=17)$, plus de $100 €(\mathrm{n}=11), N R(n=4)$.

- La possibilité de privatiser la salle (2 variables actives) : privatisation_non $(n=33)$, privatisation $(\mathrm{n}=112), N R(n=8)$.

\section{Programmation artistique de la salle}

- $\quad$ Nombre de genres musicaux joués dans la salle (ils ne sont pas forcément joués lors des mêmes concerts), parmi 13 «genres $»^{45}(3$ modalités actives) : 1 genre $(n=25), 2$ à 4 genres $(n=83), 5$ genres ou plus $(n=45)$.

- Pluridisciplinarité de la salle, caractérisée par le nombre d'autres activités présentées parmi la danse, le théâtre, l'organisation de rencontres et l'existence d'expositions, selon 3 modalités actives : pas d'autres activités $\left(0 \_\right.$pluri ; n=73) ; une à deux autres activités (MoyenPluri_1_2; n=61), trois ou quatre autres activités (FortPluri_3_4;n=19). Les projections de films n'ont pas été retenues car elles concernaient un nombre trop minoritaire de salles.

- Existence de pratiques d'amateur, qui concernent la mise à disposition de locaux de répétition, la présentation d'artistes amateurs, la proposition de formations à destination des artistes etc. (2 modalités actives) : amateurs_non $(n=123)$, amateurs $(n=30)$.

\footnotetext{
${ }^{45}$ Sont ainsi pris en compte le rock, la pop, le jazz, les musiques classiques, les musiques du monde, les variétés françaises, le hiphop, les musiques expérimentales, la funk (avec la soul), la chanson francophone, la techno, les musiques électroniques et le rap.
} 


\section{Ouverture de la salle}

- $\quad$ Ouverture le jour (2 modalités actives) : la salle est accessible en journée, avant 17 heures (« ouvert_jour »; n=63), la salle n'ouvre que le soir (fermé_jour ; n=90).

- $\quad$ Ouverture la nuit (2 modalités actives) : la salle ferme après l'événement ou au plus tard à 2 heures du matin $(2 \mathrm{H} ; \mathrm{n}=97)$, la salle est régulièrement autorisée à ouvrir au-delà de 2 heures du matin $(5 \mathrm{H} ; \mathrm{n}=56)$.

\section{Visibilité numérique}

- Présence sur le réseau social Facebook (4 modalités actives). Cette variable renseigne à la fois sur la stratégie de promotion de la salle, sur sa proximité avec les outils numériques, sur sa notoriété (Facebook est aujourd'hui relativement utilisé en France, y compris chez les plus de 35 ans), mais aussi sur la mesure dans laquelle il est socialement gratifiant d'exprimer publiquement que l'on « aime» (« like ») cette salle. Elle se fonde donc sur le nombre de personnes ayant coché « like » sur la page Facebook de la salle au $1^{\mathrm{er}}$ mai 2014 : Sans_Facebook (n=19), 0 à $10000(n=79), 10001$ à $30000(n=36)$, plus de 30000 $(n=19)$.

- Présence sur le réseau social de micro-blogging Twitter, au $1^{\mathrm{er}}$ mai 2014 (2 modalités actives). Cette variable renseigne sur la stratégie numérique de la salle, ce qui recoupe en partie sa notoriété et le type de publics auxquels elle souhaite s'adresser (en France, le taux de personnes actives sur Twitter serait d'environ 4\% des personnes utilisant internet et l'âge moyen de ses utilisateurs serait de 22 ans $\left.^{46}\right)$ : Twitter_oui $(n=82)$, Twitter_non $(n=71)$.

46 Données issues de «L'utilisateur type de Twitter, en France et dans le monde », 21.11.2013, http://www.blogdumoderateur.com/utilisateur-type-twitter/ consulté le 18.05.2014. 


\section{Spécificités de la programmation}

17 variables concernant les genres musicaux et l'internationalisation des programmations ont été mises en éléments supplémentaires. Nous ne souhaitions pas que les genres musicaux entrent en compte dans la formation de l'espace, puisqu'il importe ici de voir comment ceux-ci se répartissent en fonction des propriétés des salles. Le découpage des «genres » musicaux ici ne reprend pas exactement les catégories utilisées dans les enquêtes d'Olivier Donnat sur les pratiques culturelles des français. Effectivement, la définition des genres musicaux pose toujours question et la répartition retenue demeure imparfaite, englobant des catégories très larges : le terme « rock » recouvre aujourd'hui un nombre important d'artistes aux qualités diverses. Son hégémonie sur la scène parisienne (49\% des salles en programment) semblerait bien refléter aussi un problème de catégorisation trop étendue. Cependant, afin de ne pas trop brouiller l'analyse, nous avons choisi de retenir 16 genres (trois genres, concernant les musiques classiques, ont été ajoutés par rapport à la variable «nombre de genres musicaux joués »), adaptés à l'offre de la ville. Une salle peut donc jouer plusieurs genres, ce qui concerne la majorité des salles (seuls 16\% ne jouent qu'un genre). Nous les avons codés en consultant au moins un mois de programmation, selon les désignations utilisées par la communication de la salle lorsqu'il y en avait ou en consultant la documentation disponible à propos des artistes. Il s'agit ici d'une approche relationnelle et non substantialiste des genres musicaux, dans le sens où revendiquer l'appartenance de sa musique à tel ou tel genre participe d'un positionnement dans des luttes de définition. Les genres retenus ne se caractérisent donc pas par des critères musicaux que nous aurions élaborés.

Les variables sont toutes dichotomiques (oui, indiqué par des cercles pleins/non, indiqué par des cercles vides) : rock $(n=75)$, musiques électroniques $(n=74)$, musiques du monde (avec la world music et les musiques dites traditionnelles) $(n=64)$, chanson francophone 
$(n=56)$, jazz $(n=48)$, hiphop $(n=46)$, pop $(n=46)$, funk et soul $(n=29)$, techno $(n=27)$, musiques classiques ( $\mathrm{n}=23$ ) (qui inclut le classique, le baroque et la musique de chambre ( $n=21)$, la musique contemporaine $(n=12)$ et l'opéra, l'opérette $(n=10))$, variétés françaises ( $n=21)$, musiques expérimentales (avec la noise et le punk) (n=19), rap ( $n=16)$. Comme souvent, le regroupement en catégories effectué ici n'est pas totalement satisfaisant. Par exemple, le regroupement des salles de musiques dites expérimentales avec la noise et le punk peut sembler contre-intuitif, cependant une seule des 12 salles présentant ces deux derniers genres ne joue pas aussi des musiques expérimentales. De même, le metal, le hard rock et leurs variantes, peu audibles dans l'espace parisien, ont été inclus dans le rock.

La dernière variable est un indice d'internationalisation de la programmation de la salle (en gris). Il a été construit en consultant un mois de programmation. Le pourcentage d'artistes venus de l'étranger a été calculé (un groupe compte pour 1 artiste) : de 0 à $10 \%$ $(\mathrm{n}=68)$, de 10 à 30\% (n=34), de 31 à 50\% (n=21), de 51 à $70 \%(\mathrm{n}=13)$, plus de $70 \%(\mathrm{n}=7)$, $\operatorname{NR}(n=10)$. 


\section{Encadré 2. Les discours de présentation des lieux}

Les discours de présentation des lieux fournissent quelques exemples de la différence entre salles ancrées depuis plus longtemps dans le paysage parisien et musical, plus installées notamment grâce aux succès commerciaux ou soutiens publics (les «assis »), et d'autres récentes ou «nouvelles », existant dans un contexte plus concurrentiel, leur demandant de se distinguer afin d'être identifiées (les « debout»). La rhétorique des premières rend visible leur héritage et leur ancrage géographique, donc leur capital symbolique. Les autres lieux adoptent des stratégies de distinction par rapport au pôle dominant : des pratiques et une salle présentées comme différentes, mettant en avant la rencontre, des publics diversifiés, des artistes émergents et la référence à des ailleurs définis comme emblématiques (salles exemples sur le graphique $6)$.

Ainsi, le Théâtre des Champs Elysées ( $8^{\text {ème }}$ arr.) se met en scène de la manière suivante : «L'aventure artistique du Théâtre des Champs-Elysées est illustrée par la présence, depuis plus de 90 ans, des artistes les plus prestigieux de l'histoire de la musique, de l'opéra et de la danse ${ }^{47}$, alors que le Caveau de la Huchette ( $5^{\text {ème }}$ arr.), « le temple du swing depuis 65 ans », annonce « Chaque soir un orchestre de jazz pour le plaisir d'écouter ou de danser, dans la pure tradition de l'époque des Cotton club, Savoy... $»^{48}$. Quant au cabaret Chez Michou (18 ème arr.), il précise «Le dernier dinosaure de la nuit parisienne a débarqué Gare du Nord venant d'Amiens, sa ville natale. La rue de Dunkerque puis la place Blanche et la rue Lepic... C'était en 1949, et il ne quittera plus Montmartre. Depuis le 13 juillet 1956 il règne sur le 80 rue des Martyrs. ${ }^{49}$ De l'autre côté de l'espace dessiné par l'ACM, se trouvent le Petit Bain (13 ${ }^{\text {ème }}$ arr.) :

\footnotetext{
${ }^{47}$ «Un peu d'histoire », http://www.theatrechampselysees.fr/le-theatre/un-peu-d-histoire, consulté le 15.05.2014

${ }^{48} \mathrm{http}: / / \mathrm{www}$. caveaudelahuchette.fr, consulté le 15.05.2014.

${ }^{49}$ http://www.michou.com/cabaret.html, consulté le 15.05.2014.
} 
« une salle de concerts... mais pas que. Petit Bain est un restaurant, mais pas que. Petit Bain c'est aussi une entreprise d'insertion par l'activité économique, une coopérative où chaque acteur a son mot à dire. C'est aussi un lieu accessible aux personnes à mobilité réduite ainsi qu'aux sourds et malentendants. Petit Bain c'est un espace pour écouter, voir, entendre, jardiner, danser, apprendre, rencontrer,... Un espace convivial pour montrer qu'il est possible d'entreprendre autrement. $»^{50}$

ou encore le Djoon (13 ${ }^{\text {ème }}$ arr.) :

« Niché dans le nouveau quartier dynamique de l'Est parisien, ce club à taille humaine a accueilli pendant 8 ans avec le même enthousiasme les talents connus ou inconnus de la scène locale et internationale, de la soulful new yorkaise à la deep afro sud africaine en passant par les pionniers de Chicago Détroit, de la deep tech berlinoise aux très éclectiques londoniens... Toujours à la recherche des talents d'aujourd'hui et de demain, ce temple de la rencontre entre danseurs et music lovers a vu son parquet foulé par des clubbers venus des quatre coins de la planète. $»^{51}$

${ }^{50} \mathrm{http}: / /$ www.petitbain.org, consulté le 15.05.2014, souligné par l'auteure.

${ }^{51}$ https://www.facebook.com/DjoonClub/info, consulté le 15.05.2014, souligné par l'auteure. 
Graphique 1. Projection des variables actives dont les modalités contribuent au-dessus de la moyenne, Axes 1 et 2

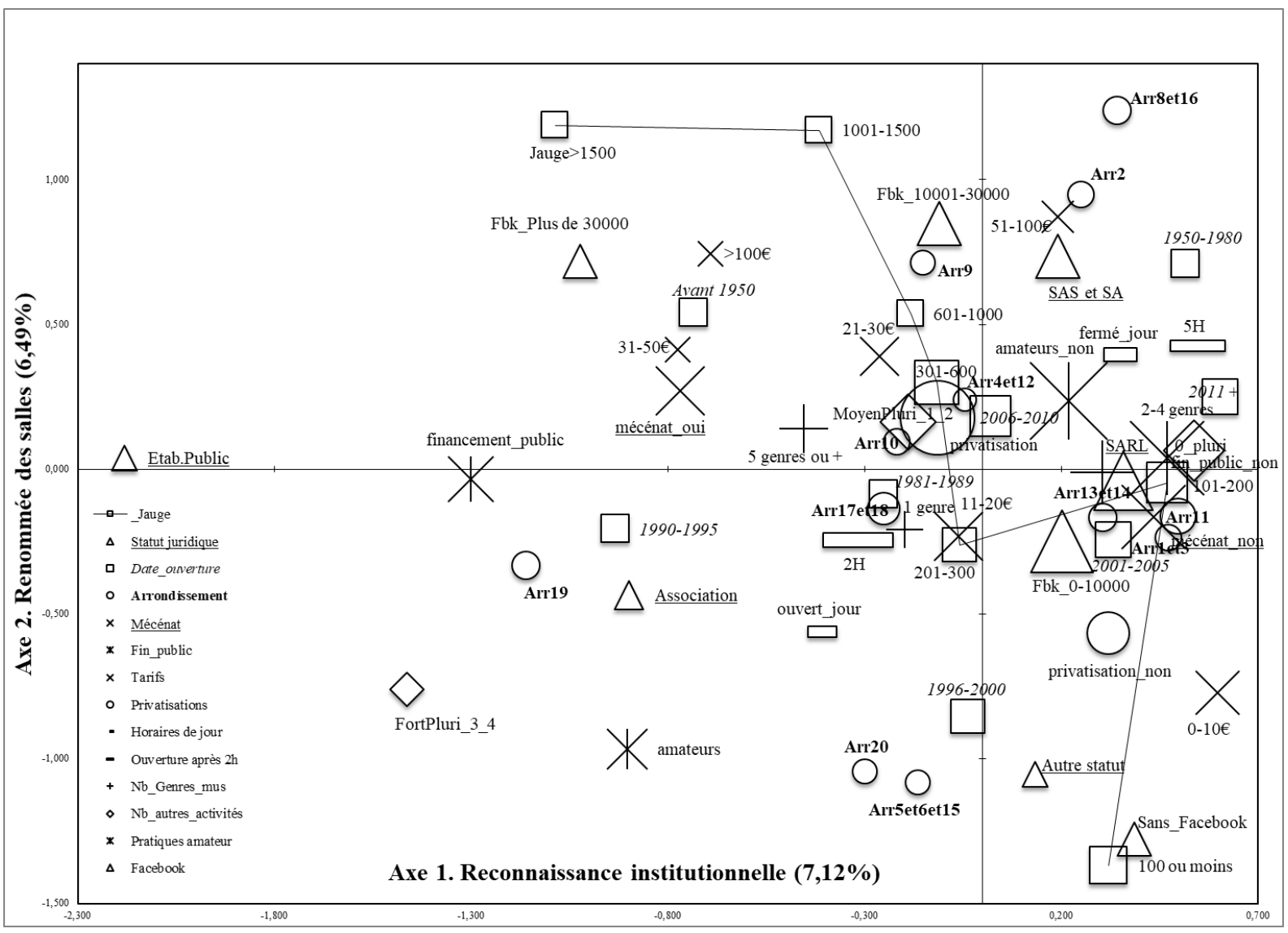

Graphique 2. Projection des variables actives dont les modalités contribuent au-dessus de la moyenne, Axes 2 et 3

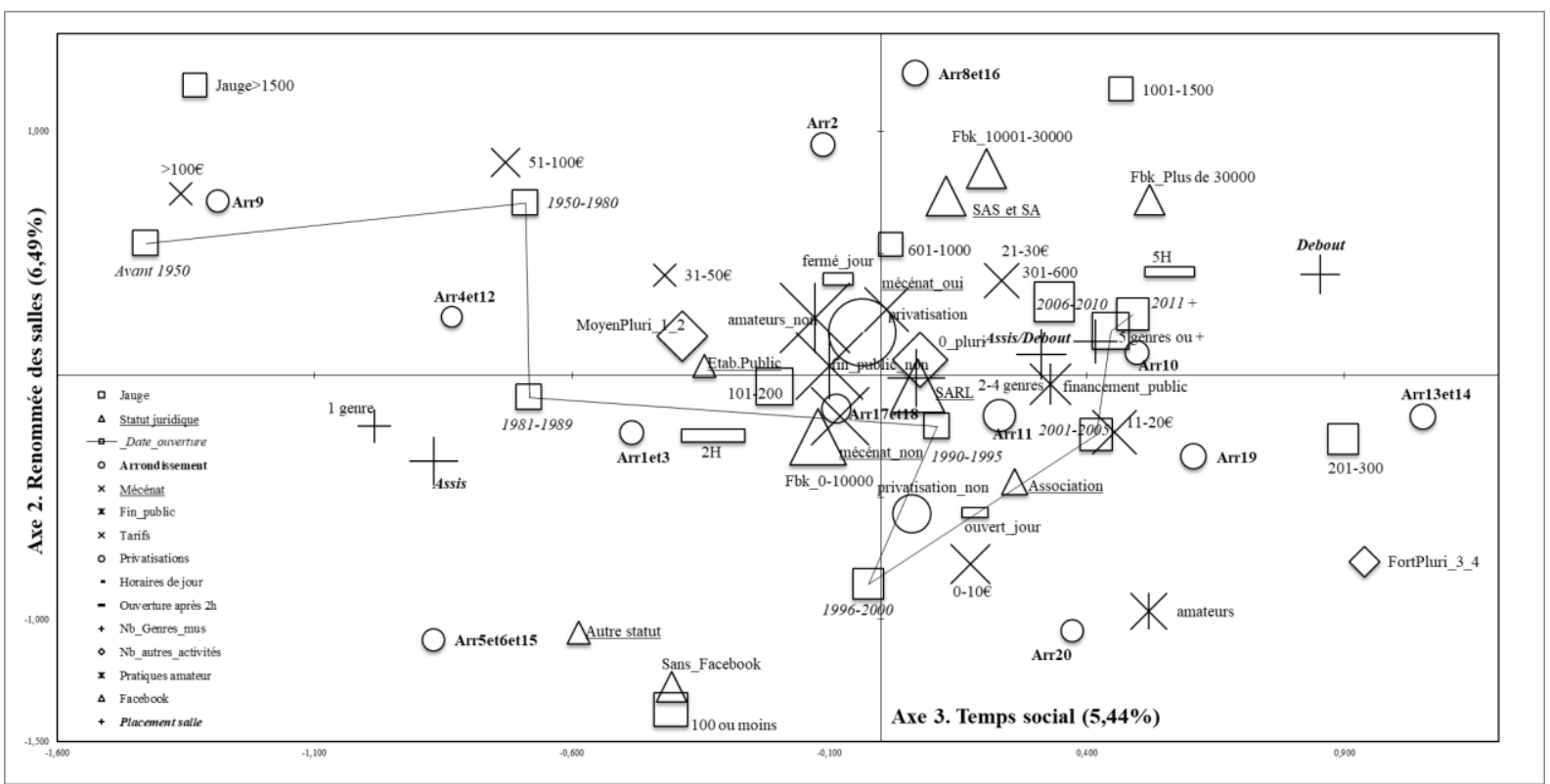


Graphique 3. Projection des variables supplémentaires, Axes 1 et 2

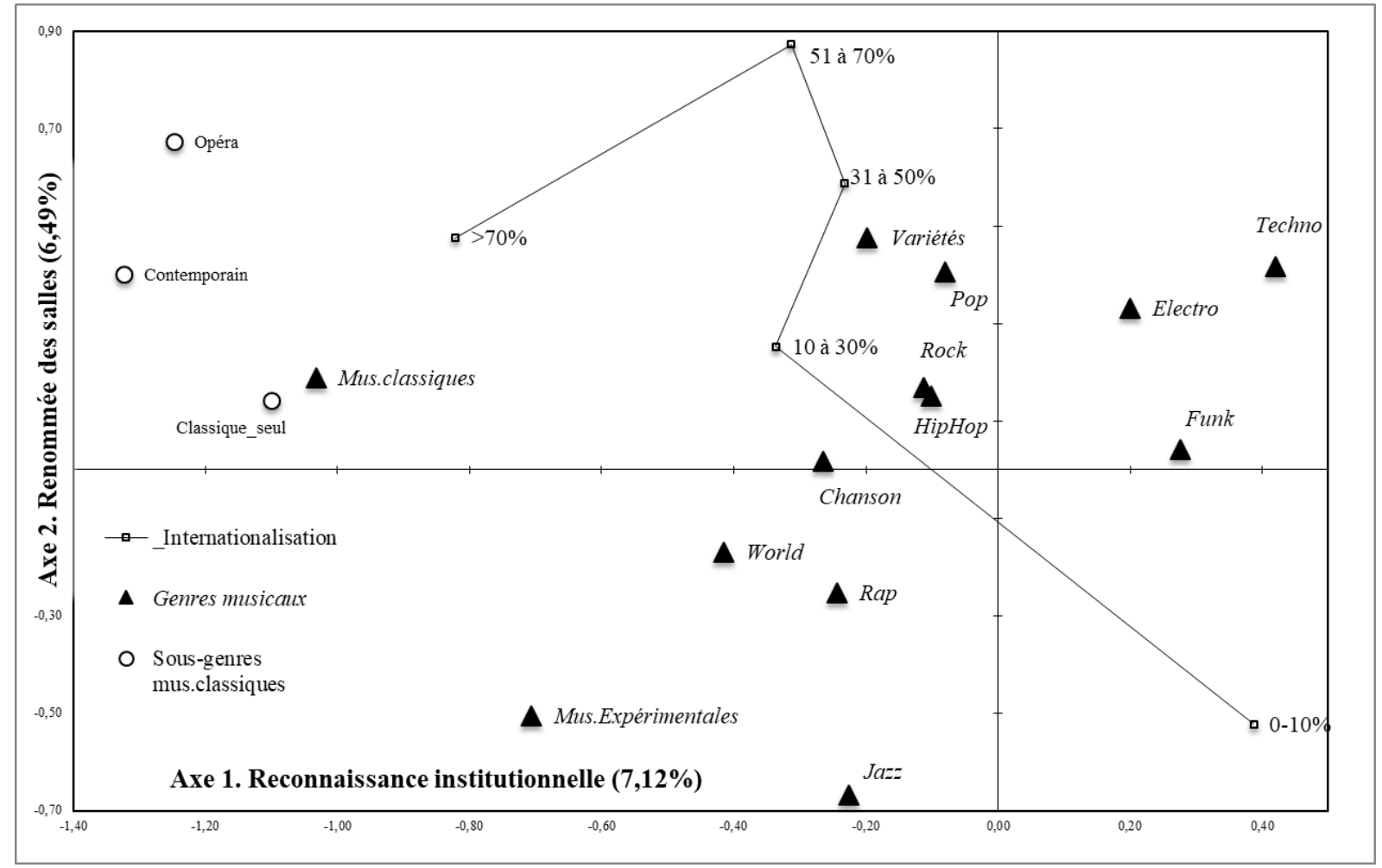

Graphique 4. Projection des variables supplémentaires, Axes 2 et 3

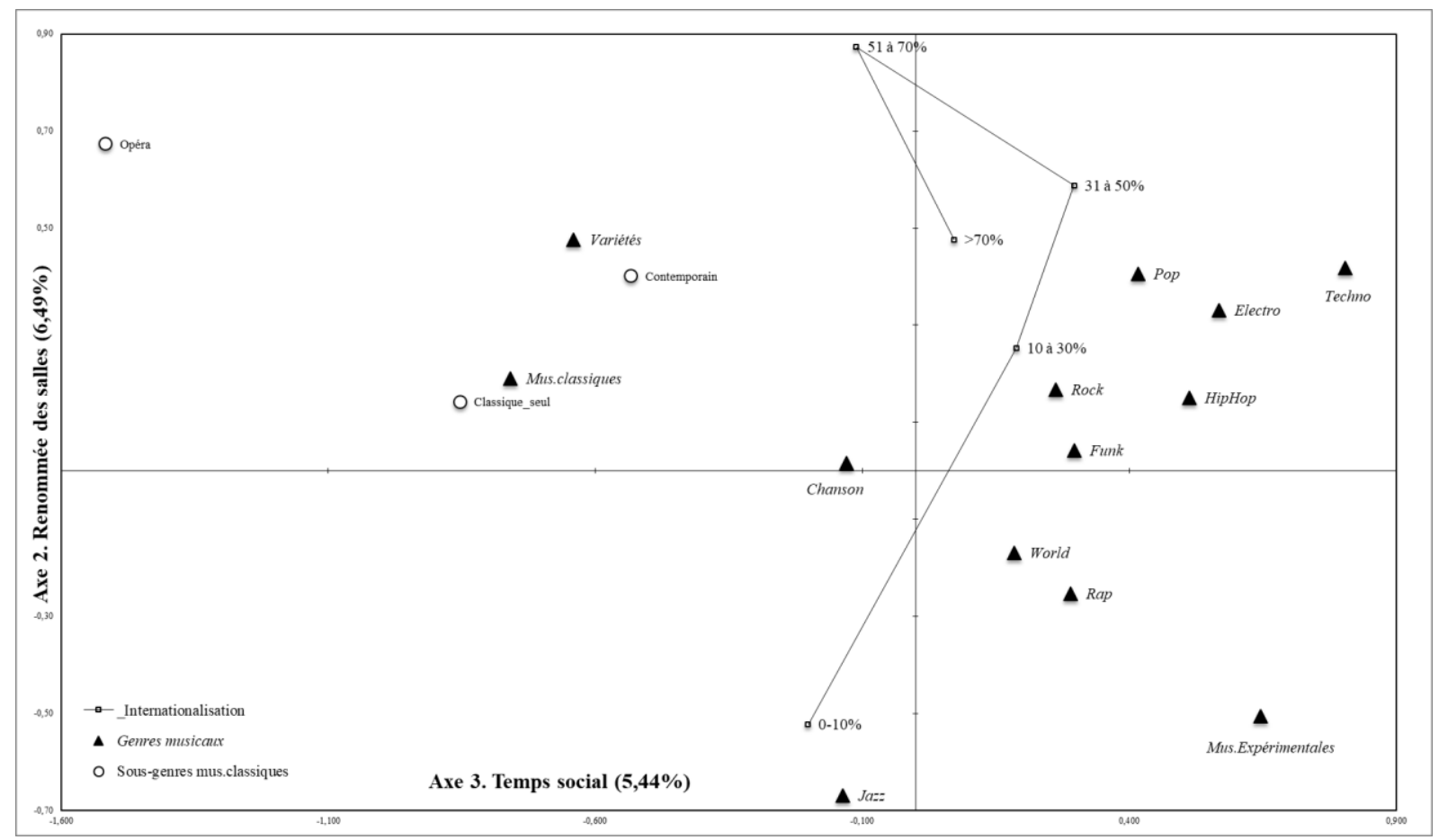


Graphique 5. Nuage des individus actifs dans le plan des axes 1 et 2

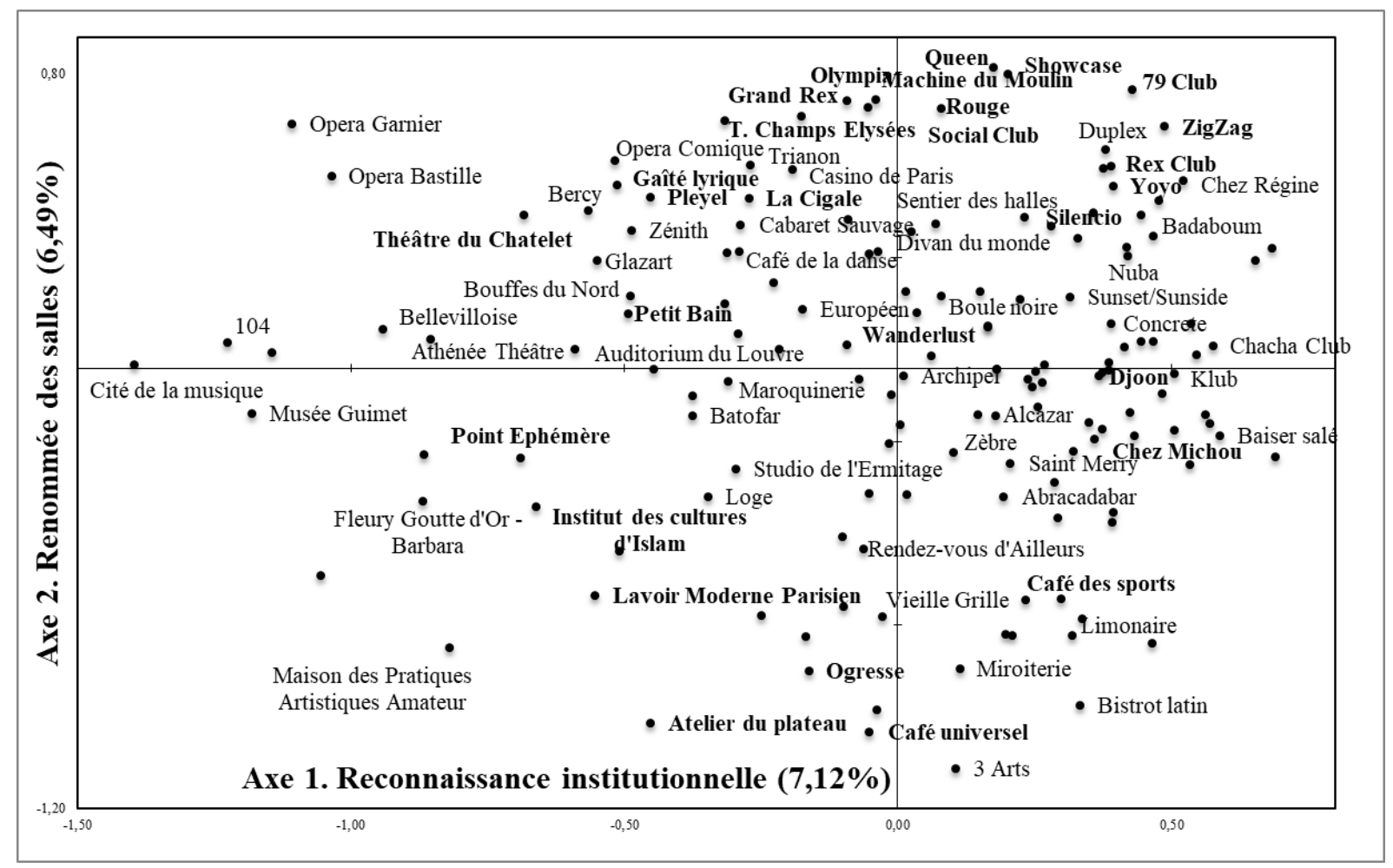

Graphique 6. Nuage des individus actifs dans le plan des axes 2 et 3

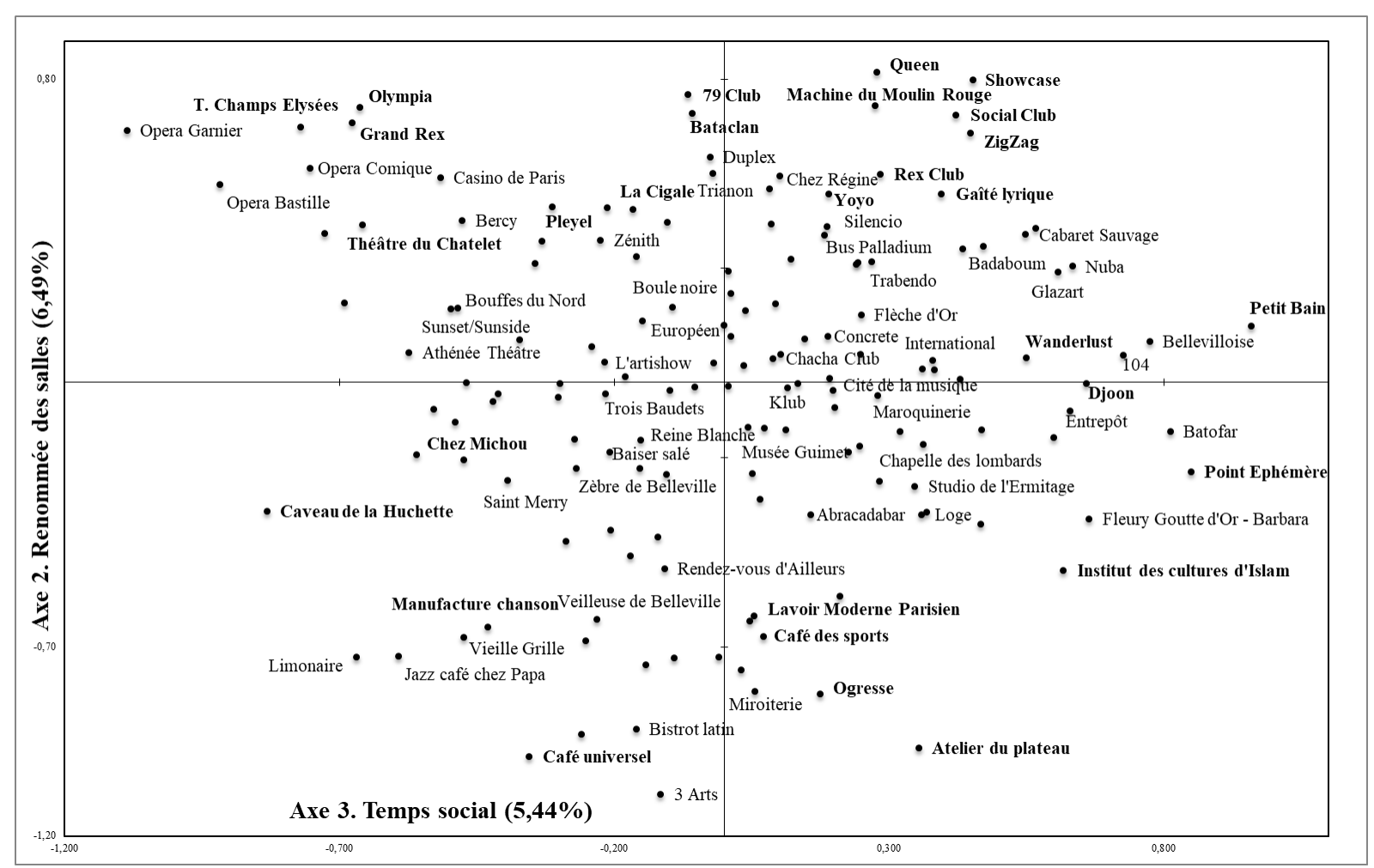

